\title{
Natural three-valued logics and classical logic
}

Natalya E. TOMOVA

\begin{abstract}
In this paper implicative fragments of natural threevalued logic are investigated. It is proved that some fragments are equivalent by set of tautologies to implicative fragment of classical logic. It is also shown that some natural three-valued logics verify all tautologies of classical propositional logic.

Keywords: three-valued logis, natural implication, classical logic, set of tautologies
\end{abstract}

\section{Introduction}

In paper [3] we investigated functional properties of three-valued logics. We define some conditions for 'good' implication and introduce the idea of natural implication. So, as the result we have class of 30 implications ${ }^{1}$ with strictly specified natural properties. Extensions of regular Kleene's logics by natural implications were regarded.

According to our definition, natural three-valued logic is a logic which includes natural implication as a connective.

On examination of 30 implicative extensions of weak Kleene's logic we received 7 basic $\operatorname{logics}^{2}$ : Łukasiewicz's logic $\mathbf{E}_{\mathbf{3}}$, paraconsistent logic PCont, three-valued Bochvar's logic $\mathbf{B}_{\mathbf{3}}, \operatorname{logic} \mathbf{Z}, \mathbf{T}^{\mathbf{3}}$, $\mathbf{T}^{\mathbf{2}}$ and $\mathbf{T}^{\mathbf{1}}$. These logics form a lattice w.r.t. relation of functional inclusion one logic to another.

Thus all these different three-valued systems, which appeared historicaly on different motivations, are presented in the same language

\footnotetext{
${ }^{1}$ Truth-tables for natural implications are given in appendix.

${ }^{2}$ In [4] the functional eqiuvality of some implicative extensions of weak
} Kleene's logic was proved. 
with the following connectives: $\sim, \cup$ and $\rightarrow$, where $\sim, \cup-$ connectives of weak Kleene's logic and $\rightarrow-$ natural implication. It will allow us to compare these logics by set of tautologies. This is the next point of our research. And in this paper we focus on implicative fragments of natural three-valued logics.

\section{Basic definitions}

For the sake of clarity let us formulate some basic definitions.

DeFinition 1 . The language $L_{\rightarrow}$ is a propositional language with the following alphabet:

(1) $p, q, r, \ldots$ - propositional variables;

(2) $\rightarrow$ - binary logical connective;

(3) $($,$) - technical symbols.$

Definition 2. A definition of $L_{\rightarrow}$-formula is as usual:

(1) if $A$ is propositional variable, then $A$ is $L_{\rightarrow}$-formula;

(2) if $A$ and $B$ are $L_{\rightarrow}$-formulas, then $A \rightarrow B$ is $L_{\rightarrow}$-formula;

(3) nothing else is $L_{\rightarrow}$-formula.

Definition 3. A logical matrix is a structure $\mathfrak{M}=\langle V, F, D\rangle$, where $V$ is the set of truth-values, $F$ is a set of functions on $V$ called basic functions, and $D$ is a set of designated values, $D$ is a subset of $V$.

In this paper we will concider the logical matrices, where $V=$ $\{1,1 / 2,0\}$ (let denote this set as $V_{3}$ ), $F$ consists of one function ${ }^{3}-$ natural implication and $D=\{1\}$ or $D=\{1,1 / 2\}$.

Let's recall definition of natural implication:

DEFINITION 4. Implication is called natural if it is satisfied the following criteria:

(1) $\mathbf{C}$-extending, i.e. restrictions to the subset $\{0,1\}$ of $V_{3}$ coincide with the classical implication.

\footnotetext{
${ }^{3}$ When we consider the implicative fragments of natural three-valued logics.
} 
(2) If $p \rightarrow q \in D$ and $p \in D$, then $q \in D$, i.e. matrices for implication need to be normal in the sense of LukasiewiczTarski (they verify the modus ponens) [2, p. 134].

(3) Let $p \leq q$, then $p \rightarrow q \in D$.

(4) $p \rightarrow q \in V_{3}$, in other cases.

According to the definition of natural implication, there are 6 implications with $D=\{1\}$ and 24 implications with $D=\{1,1 / 2\}$ (appropriate truth-tables are given in appendix).

Definition 5. A valuation $v$ of an arbitrary $L_{\rightarrow}$-formula $A$ in $\mathfrak{M}$ (symbolically $-|A|_{v}^{\mathfrak{M}}$ ) is defined as usual: $|p|_{v}^{\mathfrak{M}} \in V_{3}$, if $p$ is a propositional variable; if $A$ and $B$ are $L_{\rightarrow}$-formulas, and $\rightarrow$ is basic function in $\mathfrak{M}$, then $|A \rightarrow B|_{v}^{\mathfrak{M}}=|A|_{v}^{\mathfrak{M}} \rightarrow|B|_{v}^{\mathfrak{M}} \cdot{ }^{4}$

Definition 6. An arbitrary $L_{\rightarrow}$-formula $A$ is a tautologie in $\mathfrak{M}$ iff $|A|_{v}^{\mathfrak{M}} \in D$ for all valuation $v$ in $\mathfrak{M}$.

\section{Implicative fragments of natural three-valued $\operatorname{logics}$}

Let consider the following matrices which corespond to the implicative fragments of natural three-valued logics:

$$
\begin{aligned}
& \mathfrak{M}_{\rightarrow}^{i}=<\{1,1 / 2,0\}, \rightarrow_{i},\{1\}>, i \in\{1,2,3,4,5,6\}, \\
& \mathfrak{M}_{\rightarrow}^{i}=<\{1,1 / 2,0\}, \rightarrow_{i},\{1,1 / 2\}>, i \in\{7,8,9,10,11, \\
& 12,13,14,15,16,17,18,19,20,21,22,23,24,25,26,27,28\}, \\
& \mathfrak{M}_{\rightarrow}^{1^{\prime}}=<\{1,1 / 2,0\}, \rightarrow_{1},\{1,1 / 2\}>, \\
& \mathfrak{M}_{\rightarrow}^{4^{\prime}}=<\{1,1 / 2,0\}, \rightarrow_{4},\{1,1 / 2\}>,
\end{aligned}
$$

where matrix operation $\rightarrow$ is defined by appropriate truth-tables of natural implications.

The following tautologies express the fundamental properties of implication:

\footnotetext{
${ }^{4}$ For the clarity we use the same symbols both for language functor (propositional connective) and corresponding matrix function.
} 
$K: p \rightarrow(q \rightarrow p)$

$S:(p \rightarrow(q \rightarrow r)) \rightarrow((p \rightarrow q) \rightarrow(p \rightarrow r))$

$S^{\prime}:((p \rightarrow q) \rightarrow r) \rightarrow((p \rightarrow r) \rightarrow(q \rightarrow r))$

$P:((p \rightarrow q) \rightarrow p) \rightarrow p$

$W:(p \rightarrow(p \rightarrow q)) \rightarrow(p \rightarrow q)$

$C:(p \rightarrow(q \rightarrow r)) \rightarrow(q \rightarrow(p \rightarrow r))$

$B:(q \rightarrow r) \rightarrow((p \rightarrow q) \rightarrow(p \rightarrow r))$

And all implicative fragments of natural three-valed logics can be divided into 10 classes according to the fact that implicative formulas are tautologies in corresponding matrices:

\begin{tabular}{|c|c|c|c|c|c|c|c|}
\hline & $K$ & $S$ & $S^{\prime}$ & $P$ & $W$ & $C$ & $B$ \\
\hline $\begin{array}{c}\mathfrak{M}_{\rightarrow}^{1^{\prime}}, \mathfrak{M}_{\rightarrow}^{i} \\
(i \in\{2,5,7,8,9,10,11,12,13 \\
17,18,19,20,21,22,23,24\})\end{array}$ & + & + & + & + & + & + & + \\
\hline $\mathfrak{M}_{\rightarrow}^{1}$ & + & + & + & - & + & + & + \\
\hline $\mathfrak{M}_{\rightarrow}^{25}$ & - & + & + & - & + & + & + \\
\hline $\mathfrak{M}_{\rightarrow}^{3}$ & + & - & + & - & - & + & + \\
\hline $\mathfrak{M}_{\rightarrow}^{4}, \mathfrak{M}_{\rightarrow}^{4^{\prime}}$ & - & + & + & - & + & - & + \\
\hline $\mathfrak{M}_{\rightarrow}^{27}$ & - & - & + & + & + & - & - \\
\hline $\mathfrak{M}_{\rightarrow}^{26}$ & - & + & - & - & + & - & - \\
\hline $\mathfrak{M}_{\rightarrow}^{i}(i \in\{15,28\})$ & - & - & - & + & + & - & - \\
\hline $\mathfrak{M}_{\rightarrow}^{6}$ & - & - & + & - & - & - & - \\
\hline $\mathfrak{M}_{\rightarrow}^{i}(i \in\{14,16\})$ & - & - & - & - & - & - & - \\
\hline
\end{tabular}

So, let us consider the class matrices (corresponding to the first line of table above), in which all given implicative formulas are tautologies. This class consists of 18 matrices: 2 with $D=\{1\}$ and 16 with $D=\{1,1 / 2\}$. We can prove that all these matrices have the same class of tautologies.

The reasoning is as follows. For example, consider the matrices: 


$$
\begin{aligned}
& \mathfrak{M}_{\rightarrow}^{7}=<\{1,1 / 2,0\}, \rightarrow_{7},\{1,1 / 2\}> \\
& \mathfrak{M}_{\rightarrow}^{13}=<\{1,1 / 2,0\}, \rightarrow_{13},\{1,1 / 2\}>
\end{aligned}
$$

To show that these matrices have the same class of tautologies is sufficent to prove the following theorem:

Theorem 1. For any $L_{\rightarrow}$-formula $A$, for any valuation $v^{5}$ :

$$
|A|_{v}^{\mathfrak{M}_{\rightarrow}^{7}}=0 \text { iff }|A|_{v}^{\mathfrak{M}^{13}}=0 .
$$

Proof may be given by induction on the structure of formula $A$.

Base case. Let $A$ is a propositional variable, then it is obvious that theorem is true for this case.

Induction step. Let us assume that theorem is true for the formulas, that contain less than $n$ occurrence of propsitional connectives (induction hypothesis). Then it is sufficent to prove, that theorem is true for $L_{\rightarrow}$-formula $A$ that contains precisely $n$ occurrence of propsitional connectives and graphically identical with formula $(B \rightarrow C)$, i.e. $A=(B \rightarrow C)$.

Then, the proof of the theorem reduces to the proof of the following two propositions:

Proposition 1. $\forall v \forall A:$ if $|A|_{v}^{\mathfrak{M}^{7}}=0$, then $|A|_{v}^{\mathfrak{M}^{13}}=0$.

Proposition 2. $\forall v \forall A:$ if $|A|_{v}^{\mathfrak{M}^{13}}=0$, then $|A|_{v}^{\mathfrak{M}^{7}}=0$.

Let us present the proof of the Proposition 1.

\section{ProOF.}

1. Let proposition 1 does not hold - assumption

2. $\quad \exists v \exists A:|A|_{v}^{\mathfrak{M}^{7}}=0$ and $|A|_{v}^{\mathfrak{M}^{13}} \rightarrow 0$

- from 1

3. $\quad|B \rightarrow C|_{v^{*}}^{\mathfrak{M}^{7}}=0$ and $|B \rightarrow C|_{v^{*}}^{\mathfrak{M} 13} \neq 0$

- from 2, elimination of quantifiers

4. $\quad|B \rightarrow C|_{v^{*}}^{\mathfrak{M}_{\rightarrow}^{7}}=0$

- from 3

5. $\quad|B|_{v^{*}}^{\mathfrak{M}^{7}} \rightarrow_{7}|C|_{v^{*}}^{\mathfrak{M}^{7}}=0$

6. $|B|_{v^{*}}^{\mathfrak{M}^{7}} \in\{1,1 / 2\}$ and $|C|_{v^{*}}^{\mathfrak{M}^{7}}=0$

- from 4 , def. 5

- from 5 , def. of $\rightarrow_{7}$

\footnotetext{
${ }^{5}$ As set $V_{3}$ in $\mathfrak{M}_{\rightarrow}^{7}$ and in $\mathfrak{M}_{\rightarrow}^{13}$ is the same, then it is true that any valuation in $\mathfrak{M}_{\rightarrow}^{7}$ is valuation in $\mathfrak{M}_{\rightarrow}^{13}$ and vice versa.
} 

7. $|C|_{v^{*}}^{\mathfrak{M}^{7}}=0$
- from 6
8. $|C|_{v^{*}}^{\mathfrak{M} \rightarrow 3}=0$
- from 7 by induc-
9. $\quad|B \rightarrow C|_{v^{*}}^{\mathfrak{M}^{13}} \neq 0$ tion hypothesis
10. $|B|_{v^{*}}^{\mathfrak{M}^{13}} \rightarrow 13|C|_{v^{*}}^{\mathfrak{M}^{13}} \neq 0$
- from 3
11. $|B|_{v^{*}}^{\mathfrak{M}^{13}}=0$
- from 9
12. $|B|_{v^{*}}^{\mathfrak{M}^{7}}=0$
- from 10 and 8 , def. of $\rightarrow_{13}$
12. $|B|_{v^{*}}=0$
- from 11 by induc-
13. $|B|_{v^{*}}^{\mathfrak{M}^{7}} \in\{1,1 / 2\}$ tion hypothesis
14. $|B|_{v^{*}}^{\mathfrak{M}^{7}} \neq 0$
- from 6
- from 13
15. Assumption 1. is incorrect
- from 12 and 14

Proposition 1 is proved.

The proof of Proposition 2 is analogous to that of Proposition 1. Thus theorem is proved.

By using similar methods of reasoning, it is not difficult to prove that all 18 matrices (matrices of the first group) have the same set of tautologies.

Let us investigate these 18 matrices in detail. It is well known that the implicative fragment of classical logic can be characterized deductively by the axioms $K, S$ and $P$ and the inference rule modus ponens. From this point of view each of 18 implcative fragments discussed above are the classical ones.

Let us consider natural three-valued logics, which implicative fragments are equivalent to the implicative fragment of classical logic. Corresponding logical matrices are the following:

$$
\begin{aligned}
& \mathfrak{M}^{i}=<\{1,1 / 2,0\}, \sim, \cup, \rightarrow_{i},\{1\}>, i \in\{1,5\}, \\
& \mathfrak{M}^{i}=<\{1,1 / 2,0\}, \sim, \cup, \rightarrow_{i},\{1,1 / 2\}>, \\
& i \in\{2,7,8,9,10,11,12,13,17,18,19,20,21,22,23,24\},
\end{aligned}
$$

where $\sim, \cup$ are defined like in weak Kleene's logic, appropriate truth-tables for $\rightarrow_{i}$ are given in appendix. 
From functional point of view, these 18 systems correspond to 7 basic logic:

\begin{tabular}{|c|c|c|c|c|c|c|}
\hline $\mathbf{E}_{\mathbf{3}}$ & $\mathbf{P C o n t}$ & $\mathbf{B}_{\mathbf{3}}$ & $\mathbf{Z}$ & $\mathbf{T}^{\mathbf{1}}$ & $\mathbf{T}^{\mathbf{2}}$ & $\mathbf{T}^{\mathbf{3}}$ \\
\hline $\mathfrak{M}^{i}$ & $\mathfrak{M}^{i}$ & $\mathfrak{M}^{i}$ & $\mathfrak{M}^{17}$ & $\mathfrak{M}^{23}$ & $\mathfrak{M}^{24}$ & $\mathfrak{M}^{13}$ \\
$(i \in\{1,2,8,9$, & $(i \in\{18,19$, & $(i \in\{5,7\})$ & & & & \\
$10,11,12\}$ & $20,21,22\}$ & & & & & \\
\hline
\end{tabular}

7 basic logics form the following lattice w.r.t. relation of functional inclusion one logic to another:

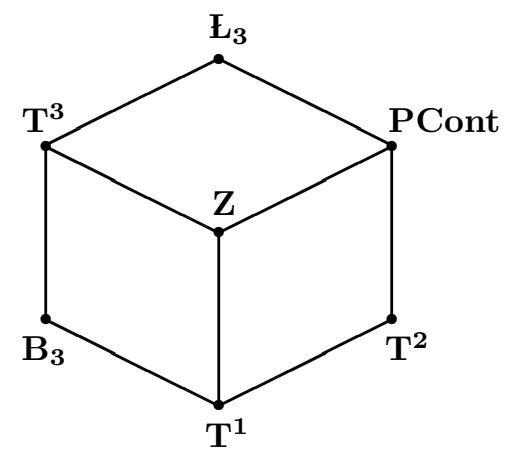

Let us show that a constant $\perp$, which interpreted as falsehood, is defined by the basic functions of the 10 matrices $\mathfrak{M}^{i},(i \in$ $\{1,2,5,7,8,9,10,11,12,13\})$ :

$$
\perp=\sim\left(p \rightarrow_{i} p\right), i \in\{1,2,5,7,8,9,10,11,12,13\} .
$$

But as follows from Wajsberg's work $[5, \S 5]$ the addition of $\perp \rightarrow p$ to the axiomatization of implicative fragment of classical logic gives the full classical propositional logic. Thus 10 natural three-valued logics, considered above, verify all tautologies of classical propositional logic.

REMARK. In [1, p. 54] by using a computer program it was calculated that there are $18 \mathrm{C}$-extending isomorphs of classical logic, which verify modus ponens. So, it was found that in matirces corresponding to these isomorphs one of the basic functions - implicative function, is defined precisely by the same truth-tables of natural implications, as in 18 natural three-valued logics mentioned above. 


\section{References}

[1] Devyatkin, L., Three-valued semantics for the classical propositional logic, Moscow: Institute of Philosophy of RAS, 2011 (in Russian).

[2] LuKAsiewiCZ, J., and A. TARski, Investigations into the sentential calculus, in Łukasiewicz J. Selected Works. Amsterdam \& Warszawa: North-Holland \& PWN. 1970.

[3] Tomova, N., A lattice of implicative extensions of regular Kleene's logics, Reports on mathematical logic 47:173-182, 2012.

[4] Tomova, N., Implicative extensions of regular Kleene's logics, Logical Investigations 16:233-258, 2010 (in Russian).

[5] Wajsberg, M., Metalogische Beiträge, Wiadomości Matematyczne 43: 131-168, 1937. (English translation: WAJsberg, M., Contribution to metalogic, Logical works, Wrocłlaw, 1977, pp. 172-200).

\section{Appendix. Truth-tables for natural implications}

Let us give truth-tables for natural implications according to the definition 4 .

There are 6 implications with $D=\{1\}$ and 24 implications with $D=\{1,1 / 2\}$. Note, that 2 paires of implications $\left(\rightarrow_{1}\right.$ and $\rightarrow_{4}$ in the proposed list below) are the same with $D=\{1\}$ and $D=\{1,1 / 2\}$. $D=\{1\}$

\begin{tabular}{|c|ccc|}
\hline$\rightarrow_{1}$ & 1 & $1 / 2$ & 0 \\
\hline 1 & 1 & $1 / 2$ & 0 \\
$1 / 2$ & 1 & 1 & 0 \\
0 & 1 & 1 & 1 \\
\hline
\end{tabular}

\begin{tabular}{|c|ccc|}
\hline$\rightarrow_{2}$ & 1 & $1 / 2$ & 0 \\
\hline 1 & 1 & $1 / 2$ & 0 \\
$1 / 2$ & 1 & 1 & 1 \\
0 & 1 & 1 & 1 \\
\hline
\end{tabular}

\begin{tabular}{|c|ccc|}
\hline$\rightarrow_{3}$ & 1 & $1 / 2$ & 0 \\
\hline 1 & 1 & $1 / 2$ & 0 \\
$1 / 2$ & 1 & 1 & $1 / 2$ \\
0 & 1 & 1 & 1 \\
\hline
\end{tabular}

\begin{tabular}{|c|ccc|}
\hline$\rightarrow_{4}$ & 1 & $1 / 2$ & 0 \\
\hline 1 & 1 & 0 & 0 \\
$1 / 2$ & 1 & 1 & 0 \\
0 & 1 & 1 & 1 \\
\hline
\end{tabular}

\begin{tabular}{|c|ccc|}
\hline$\rightarrow_{5}$ & 1 & $1 / 2$ & 0 \\
\hline 1 & 1 & 0 & 0 \\
$1 / 2$ & 1 & 1 & 1 \\
0 & 1 & 1 & 1 \\
\hline
\end{tabular}

\begin{tabular}{|c|ccc|}
\hline$\rightarrow_{6}$ & 1 & $1 / 2$ & 0 \\
\hline 1 & 1 & 0 & 0 \\
$1 / 2$ & 1 & 1 & $1 / 2$ \\
0 & 1 & 1 & 1 \\
\hline
\end{tabular}

$D=\{1,1 / 2\}$

\begin{tabular}{|c|ccc|}
\hline$\rightarrow_{7}$ & 1 & $1 / 2$ & 0 \\
\hline 1 & 1 & 1 & 0 \\
$1 / 2$ & 1 & 1 & 0 \\
0 & 1 & 1 & 1 \\
\hline
\end{tabular}

\begin{tabular}{|c|ccc|}
\hline$\rightarrow_{8}$ & 1 & $1 / 2$ & 0 \\
\hline 1 & 1 & 1 & 0 \\
$1 / 2$ & $1 / 2$ & 1 & 0 \\
0 & 1 & 1 & 1 \\
\hline
\end{tabular}

\begin{tabular}{|c|ccc|}
\hline$\rightarrow_{9}$ & 1 & $1 / 2$ & 0 \\
\hline 1 & 1 & 1 & 0 \\
$1 / 2$ & $1 / 2$ & 1 & 0 \\
0 & 1 & $1 / 2$ & 1 \\
\hline
\end{tabular}




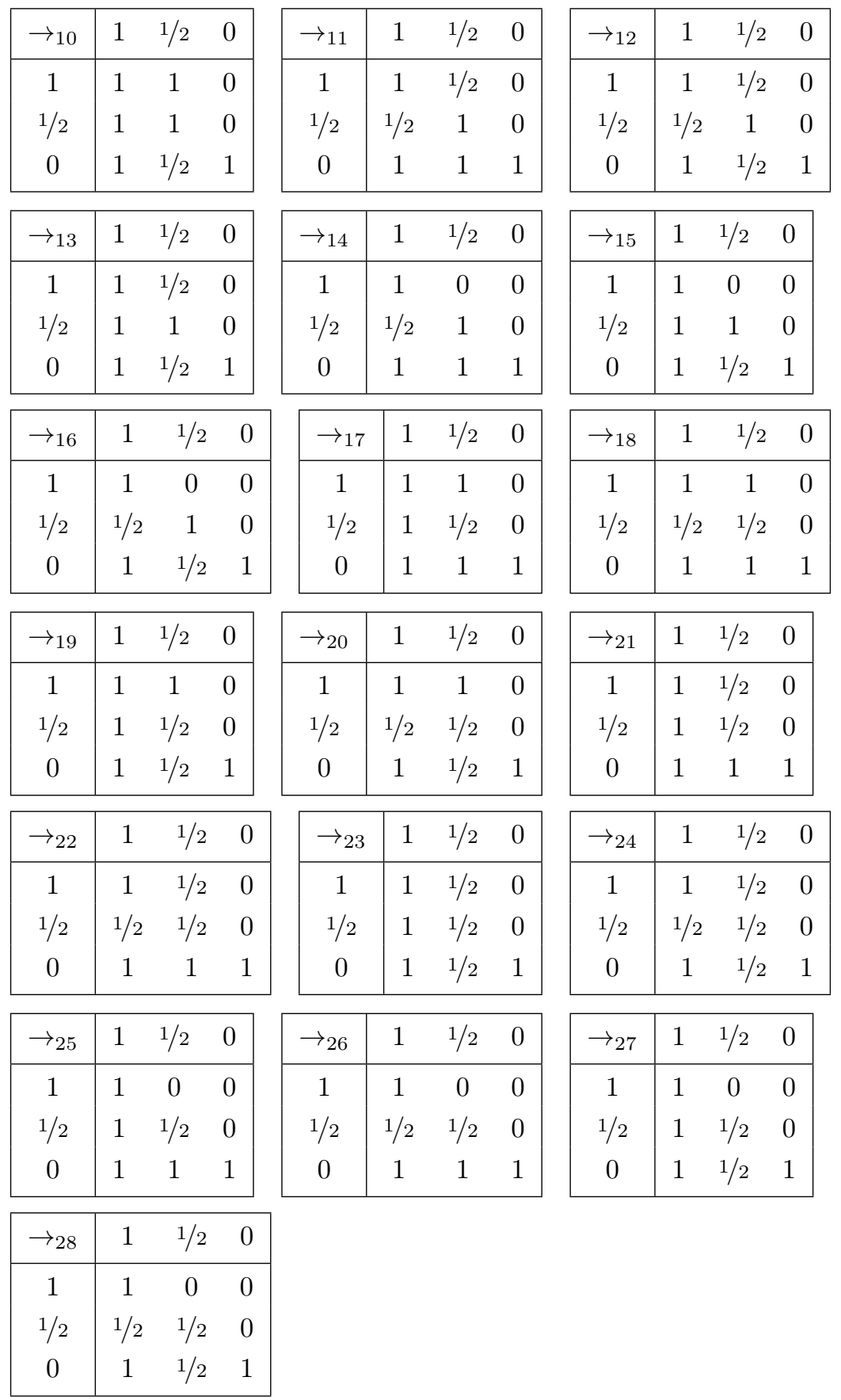

